\title{
Cardioprotective effects of anisodamine against myocardial ischemia/reperfusion injury through the inhibition of oxidative stress, inflammation and apoptosis
}

\author{
BAO-JU YAO ${ }^{1 *}$, XIAO-QING HE ${ }^{2 *}, Y^{*}-H U I L^{2}$ and WEN-JUN DAI ${ }^{2}$ \\ ${ }^{1}$ Department of Cardiology, The First People's Hospital of Huainan, Huainan, Anhui 232007; ${ }^{2}$ Department of Cardiology, \\ The Third Affiliated Hospital of Guangzhou Medical University, Guangzhou, Guangdong 510150, P.R. China
}

Received November 26, 2016; Accepted July 12, 2017

DOI: $10.3892 / \mathrm{mmr} .2017 .8009$

\begin{abstract}
The aim of the present study was to investigate the cardioprotective effects of anisodamine against myocardial ischemia/reperfusion (I/R) injury and the molecular mechanisms involved. The present results demonstrated that anisodamine attenuated myocardial infarct sizes, decreased the levels of creatine kinase and lactate dehydrogenase, whereas it increased the left ventricular (LV) systolic pressure, the LV end-diastolic pressure, and the LV pressure maximum rising and falling rates in a myocardial I/R rat model. In addition, anisodamine was revealed to suppress oxidative stress, inflammatory factor production and myocardial cell apoptosis, as demonstrated by the downregulation of caspase- 3 and apoptosis regulator BAX protein expression. The production of reactive oxygen species was decreased and the protein expression of inducible nitric oxide synthase (iNOS) was downregulated, whereas the expression of endothelial NOS was enhanced. In addition, the activity of nicotinamide-adenine dinucleotide phosphate oxidase (Nox) was suppressed and the expression of Nox4 was downregulated in rats with myocardial I/R injury. In conclusion, the results of the present study suggested that anisodamine exerted a cardioprotective effect against myocardial I/R injury in rats, through the inhibition of oxidative stress, the suppression of inflammatory processes and the inhibition of myocardial cell apoptosis.
\end{abstract}

\section{Introduction}

As a result of current lifestyle and dietary habits, the morbidity of atherosclerotic coronary heart disease (CHD) is constantly

Correspondence to: Dr Xiao-Qing He, Department of Cardiology, The Third Affiliated Hospital of Guangzhou Medical University, Liwan, 63 Duobao Road, Guangzhou, Guangdong 510150, P.R. China E-mail: qlhw3943781@126.com

*Contributed equally

Key words: anisodamine, myocardial ischemia/reperfusion, oxidative stress, inflammation, apoptosis increasing, placing CHD among the most serious threats to human health worldwide (1). In 2008, in the Global Health Statistical Report of the World Health Organization (WHO), cardiovascular and cerebrovascular diseases were reported to be the second leading cause of human mortality, following malignant tumors (2). In May 2014, the WHO updated the evaluation report regarding global health, indicating that ischemic heart disease, apoplexy, chronic obstructive pulmonary disease and lower respiratory infection are the four major causes of human mortality (3). Globally, $\sim 7$ million people succumb to ischemic heart disease and $\sim 50 \%$ of patients succumb to myocardial ischemia/reperfusion (I/R) injury. The morbidity of myocardial I/R injury increases every year, thus posing a serious threat to global health and making myocardial infarction one of the most important public health concerns currently (4).

Apoptosis is a type of programmed cell death; it is a highly-regulated process involving specific mechanisms, that is activated in mammalian cells under certain conditions. During apoptosis, endogenous DNA-degrading enzymes are activated resulting in cell death (5). Somatic cell death is a result of necrosis or apoptosis (6); these two types of cell death differ in their functions, molecular mechanisms and biochemistry.

Under ischemic conditions, the enzymatic activity of myocardial cells is severely compromised; as a result, during reperfusion, the restoration of oxygen supply further damages myocardial cells (7). During reperfusion, the generation of reactive oxygen species (ROS) is enhanced (7). ROS can destroy the structure of cell membranes through lipid peroxidation (8), thus causing an increase in membrane permeability, resulting in cellular edema. The structure of the mitochondrial membrane is also compromised, thus damaging mitochondrial function and impairing energy production, further aggravating cellular injury. ROS can oxidize proteins and impair their functions (9). When membrane protein functions are impaired, the ion exchange processes are inhibited; this is one of the primary causes of intracellular $\mathrm{Ca}^{2+}$ overload. ROS promote the chemotaxis of white blood cells; they also activate various enzymes and promote the release of prostaglandins and leukotrienes, thus further aggravating tissue damage (9). In addition, ROS can destroy nucleic acids and chromosomes; the 
resulting DNA damage also activates the pathways leading to cell apoptosis or necrosis, thus further promoting myocardial I/R injury (10).

Inflammatory processes are among the most important mechanisms implicated in the pathophysiology of myocardial I/R injury. During inflammatory reactions, the infiltration of polymorphonuclear neutrophils (PMNs) is critical (11). In I/R injury, microvascular endothelial cells and the blood vessel endothelium are damaged, attracting the adhesion of PMNs in the endothelial tissue (12). The inflammatory reaction is initiated by PMNs attachment to the blood vessel endothelium (12). This results in the increased generation of oxygen free radicals and the activation of various proteases, thus altering vascular permeability $(13,14)$. Once activated, PMNs change shape, making it difficult to penetrate through the tissues; thus, they are concentrated in the blood vessel endothelium, resulting in tissue damage and endothelial dysfunction $(12,13)$. Activated PMBs also secrete proinflammatory cytokines, including interleukin (IL)-1, IL-6 and tumor necrosis factor (TNF)- $\alpha$. Cytokines further activate the inflammatory response through nuclear factor- $\kappa \mathrm{B}$ signaling, potentiating the release of inflammatory mediators, thus forming a vicious cycle, and promoting the development and progression of I/R injury (12).

Anisodamine is a cholinergic and $\alpha_{1}$ adrenergic receptor antagonist, which has been used in China for the treatment of coronary heart disease, for the prevention and treatment of angina, pulmonary hypertension and chronic heart failure, in combination with cardiac glycosides or diuretic agents $(15,16)$. The present study aimed to investigate the putative cardioprotective effects of anisodamine against myocardial I/R injury, and to explore the underlying molecular mechanisms, using an in vivo rat model of $\mathrm{I} / \mathrm{R}$ injury.

\section{Materials and methods}

Animal experiments and ethical approval. Adult male Sprague-Dawley rats ( $\mathrm{n}=24$; weight, 220-250 g; 8-10 weeks) were provided by the Experimental Animal Center of the Third Affiliated Hospital of Guangzhou Medical University (Guangzhou, China). All experimental protocols used in the present study were approved by Committee on Animal Research and Ethics of Guangzhou Medical University (Guangzhou, China). The rats were maintained in a temperature-controlled facility, at $22 \pm 2^{\circ} \mathrm{C}$, with a relative humidity of $55 \pm 10 \%$, under a 12 -h light/dark cycle, and food and water ad libitum.

Myocardial $I / R$. All rats were randomly distributed into three groups (8 rats/group): Control group (sham-operated group); MI/R group (myocardial ischemia/reperfusion injury group); and ANI group (anisodamine treatment group). Rats were anesthetized with sodium pentobarbital $(30 \mathrm{mg} / \mathrm{kg}$, intraperitoneally), and the following surgical procedure was performed: The trachea was cannulated with a PE-90 catheter and rats were ventilated with $\mathrm{O}_{2}$ and $\mathrm{CO}_{2}$, maintaining a tidal volume of $0.8-1.2 \mathrm{ml}$. Following a left thoracotomy, the left anterior descending was exposed and occluded by ligation with a 5-0 silk suture. Myocardial ischemia was maintained for $1 \mathrm{~h}$, then the ligation was removed, and rats were reperfused for $2 \mathrm{~h}$ and treated with anisodamine. In control group, rat was anesthetized with sodium pentobarbital $(30 \mathrm{mg} / \mathrm{kg}$, intraperitoneally) and the surgical procedure was performed without ligation. In anisodamine treatment group, the rats were gavaged with $25 \mathrm{mg} / \mathrm{kg}$ of anisodamine (Shanghai Xinyijinzhu Pharmaceutical Co., Ltd., Shanghai, China) for 7 days. In sham group, rat was only anesthetized with sodium pentobarbital (30 mg/kg, intraperitoneally).

Myocardial infarct size. Hearts were harvested following treatment with anisodamine for 7 days and washed 3 times with normal saline. Heart tissue was fixed with 5\% paraformaldehyde for $24 \mathrm{~h}$ and sliced into 1-mm transverse sections and stained with 1\% 2,3,5-triphenyltetrazolium chloride at room temperature for $1 \mathrm{~h}$. Subsequently, infarct sizes were observed using inverted microscope (Leica Microsystems $\mathrm{GmbH}$, Wetzlar, Germany) and analyzed using ImageJ software version 1.43 (National Institutes of Health, Bethesda, MD, USA). Necrotic area was the white area and area at risk was determined as white area/total area $\mathrm{x} 100 \%$.

Assessment of cardiac function. Following anesthesia, the rats were secured in a supine position, an indwelling arterial needle was inserted into the right carotid artery, and the BL-420 Biological data acquisition and analysis system (Chengdu Thaimeng Software Co., Ltd., Chengdu, China) was used to measure the left ventricular (LV) systolic pressure (LVSP) and the LV end-diastolic pressure (LVEDP). The LV pressure maximum rising and falling rates $\left(+\mathrm{dp} / \mathrm{dt}_{\max },-\mathrm{dp} / \mathrm{dt}_{\max }\right)$ were analyzed using Isoheart Software, version 1.5 (Hugo Sachs Electronic, March-Hugstetten, Germany).

ELISA assays. The levels of creatine kinase (CK), lactate dehydrogenase (LDH), proinflammatory factors and oxidative stress markers, as well as the activity of caspase-3, were assessed in serum samples isolated from rats. Following anesthesia, blood samples were acquired and serum was isolated by centrifugation at $4^{\circ} \mathrm{C}$ for $10 \mathrm{~min}$ at $10,000 \mathrm{x}$. The serum levels of CK (A032) and LDH (A020-2), of the endogenous antioxidative enzyme superoxide dismutase (SOD, A001-3), of the lipid peroxidation product malondialdehyde (MDA, A003-1) and ROS (E004), of the inflammatory factors TNF- $\alpha$ (H052) and IL-6 (H007), Nox (A116) and the activity of caspase-3 (G015) were determined using commercially available ELISA kits (Nanjing Jiancheng Bio-Engineering Institute Co., Ltd., Nanjing, China).

Western blot analysis. Heart tissue samples were washed with normal saline and lysed in radioimmunoprecipitation assay lysis buffer (RIPA; EMD Millipore, Billerica, MA, USA). The supernatants were harvested by centrifugation at $4^{\circ} \mathrm{C}$ for $10 \mathrm{~min}$ at $10,000 \mathrm{x}$. The protein concentration was determined using a bicinchoninic acid protein assay kit (EMD Millipore). Equal amounts $(50 \mu \mathrm{g})$ of extracted protein samples were separated by $6-12 \%$ SDS-PAGE, depending on molecular mass, and transferred onto polyvinylidene difluoride membranes (EMD Millipore). Membranes were blocked at room temperature with 5\% fat-free milk in TBS containing Tween-20 (TBST) for $1 \mathrm{~h}$ and then incubated overnight at $4^{\circ} \mathrm{C}$ with the following primary antibodies: Anti-apoptosis regulator Bcl-2-like protein 4 (Bax; sc493; 1:500; Santa Cruz Biotechnology, Inc., 
Dallas, TX, USA), anti-B-cell lymphoma 2 (Bcl-2; sc783; 1:500; Santa Cruz Biotechnology, Inc.), anti-inducible (i)nitric oxide synthase (iNOS; sc49055; 1:500; Santa Cruz Biotechnology, Inc.), anti-endothelial (e)NOS, anti-nicotinamide-adenine dinucleotide phosphate (NADPH) oxidase 4 (Nox4; ab109225; 1:2,000; Abcam, Cambridge, UK) and anti-GAPDH (1:500, sc-25778; Santa Cruz Biotechnology, Inc.). The membranes were washed 3 times with TBST and then incubated with goat anti-rabbit horseradish peroxidase-conjugated secondary antibody (sc-2004, 1:1,000; Santa Cruz Biotechnology, Inc.) at room temperature for $1 \mathrm{~h}$. Protein bands were visualized using an enhanced chemiluminescence kit (ECL; Thermo Fisher Scientific, Inc., Waltham, MA, USA). Blots were semi-quantified using an image analyzer (Bio-Rad Laboratories, Inc., Hercules, CA, USA) and Image Lab version 3.0 (Bio-Rad Laboratories, Inc.).

Statistical analysis. Data are expressed as the mean \pm standard deviation using SPSS version 17.0 (SPSS, Inc., Chicago, IL, USA). The statistical significance of the differences between groups was assessed by one-way analysis of variance (ANOVA) followed by a post hoc Student-Newman-Keuls test for multiple comparisons. $\mathrm{P}<0.05$ was considered to indicate a statistically significant difference.

\section{Results}

Effects of anisodamine on myocardial infarct size. The effects of anisodamine on myocardial infarct sizes were investigated using a rat model of myocardial I/R. As presented in Fig. 1, myocardial infarct sizes in rats in the $\mathrm{I} / \mathrm{R}$ model group were significantly increased compared with in rats of the control group. However, treatment with anisodamine was revealed to significantly limit the size of the infarcted area in rat hearts compared with the I/R model group (Fig. 1).

Effects of anisodamine on $C K$ and $L D H$. The effects of anisodamine on the levels of $\mathrm{CK}$ and $\mathrm{LDH}$ were investigated in serum samples isolated from I/R model rats. The present results demonstrated a significant increase in CK and LDH levels in rats from the myocardial I/R model group compared with in rats in the control group (Fig. 2). Notably, treatment with anisodamine significantly suppressed the I/R-induced increase in CK and LDH serum levels compared the I/R model group (Fig. 2).

Effects of anisodamine on cardiac function. The effects of anisodamine on cardiac function were investigated in rats following myocardial I/R. As presented in Fig. 3, a significant decrease in LVSP, $+\mathrm{dp} / \mathrm{dt}_{\max }$ and $-\mathrm{dp} / \mathrm{dt}_{\max }$ was observed in rats of the I/R model group, whereas LVEDP was significantly increased compared with in the control group. Treatment with anisodamine was revealed to attenuate the I/R-induced alterations in all parameters of cardiac function: LVSP, $+\mathrm{dp}^{\mathrm{ddt}} \mathrm{t}_{\max }$ and $-\mathrm{dp} / \mathrm{dt}_{\max }$ were significantly increased, whereas the increase in LVEDP was significantly suppressed following anisodamine administration compared with rats in the I/R model group (Fig. 3).

Effects of anisodamine on the production of inflammatory factors. Compared with in rats of the control group, TNF- $\alpha$ and IL-6 levels in serum samples isolated from I/R rats were

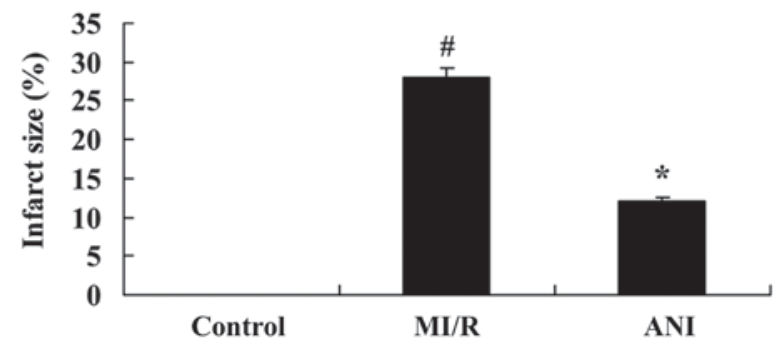

Figure 1. Effects of anisodamine on myocardial infarct size. Treatment with anisodamine significantly limited the size of the infarcted myocardium compared with the I/R model group. Data are expressed as the mean \pm standard deviation. ${ }^{\text {}} \mathrm{P}<0.05$ vs. the control group; ${ }^{*} \mathrm{P}<0.05$ vs. the MI/R group. $\mathrm{MI} / \mathrm{R}$, myocardial ischemia/reperfusion; ANI, anisodamine.

revealed to be significantly upregulated (Fig. 4). Notably, treatment with anisodamine was demonstrated to significantly attenuate the I/R-induced increase in the serum levels of TNF- $\alpha$ and IL-6 in rats compared with the I/R model group (Fig. 4).

Effects of anisodamine on oxidative stress. In order to investigate the putative antioxidative properties of anisodamine during I/R injury, the levels of SOD and MDA were measured in serum samples isolated from rats following $\mathrm{I} / \mathrm{R}$ using ELISA. As presented in Fig. 5, a significant downregulation in the levels of SOD, and an increase in MDA levels was detected in rats from the $\mathrm{I} / \mathrm{R}$ model group compared with in the control group. However, treatment with anisodamine was revealed to reverse the I/R-induced alterations, as SOD serum levels were upregulated and MDA levels were decreased following anisodamine administration compared with in untreated I/R model rats (Fig. 5).

Effects of anisodamine on caspase-3 serum levels. To further investigate the effects of anisodamine on the apoptosis of myocardial cells following I/R injury, the levels of the proapoptotic protein caspase- 3 were measured in serum samples using an ELISA kit. As demonstrated in Fig. 6, caspase-3 serum levels were significantly upregulated in rats following myocardial I/R compared with the control group. However, anisodamine administration was revealed to significantly suppress the increase in caspase- 3 levels compared with in untreated rats of the I/R group (Fig. 6).

Effects of anisodamine on ROS generation. As presented in Fig. 7, ROS levels in serum samples from I/R model rats were significantly increased compared with in control rats. The I/R-induced increase in ROS generation was revealed to be significantly suppressed following anisodamine treatment compared with in untreated rats from the I/R model group (Fig. 7).

Effects of anisodamine on apoptosis-associated protein expression. In order to investigate the effects of anisodamine on the mechanisms of myocardial apoptosis following I/R injury in vivo, the protein expression levels of the apoptosis-associated proteins apoptosis regulator $\mathrm{Bcl}-2$ and $\mathrm{Bax}$ were assessed using western blot analysis. The $\mathrm{Bax} / \mathrm{Bcl}-2$ ratio in rats from the I/R model group was significantly increased compared with in control rats (Fig. 8). Notably, treatment with 
A

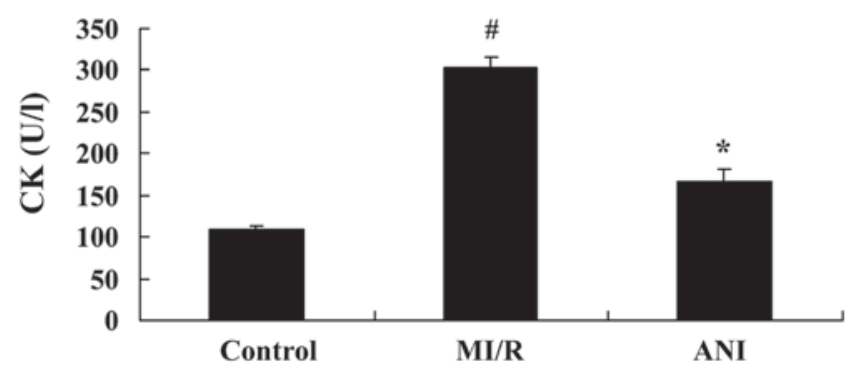

B

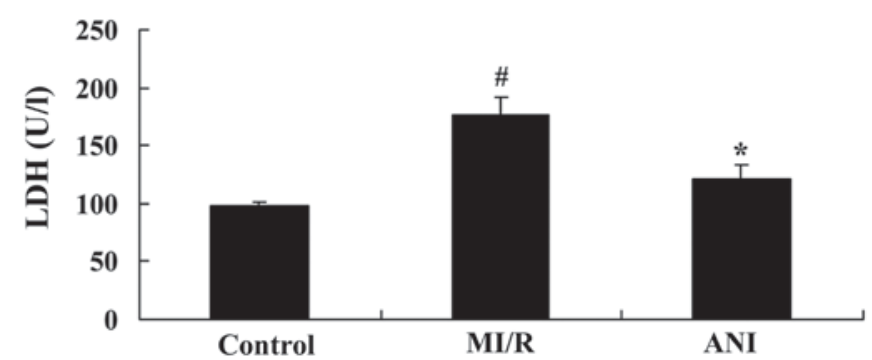

Figure 2. Effects of anisodamine on CK and LDH serum levels. Treatment with anisodamine significantly suppressed the I/R-induced increase in (A) CK and (B) LDH serum levels compared the $\mathrm{I} / \mathrm{R}$ model group. Data are expressed as the mean \pm standard deviation. ${ }^{\#} \mathrm{P}<0.05 \mathrm{vs}$. the control group; ${ }^{*} \mathrm{P}<0.05 \mathrm{vs}$. the $\mathrm{MI} / \mathrm{R}$ group. CK, creatine kinase; LDH, lactate dehydrogenase; MI/R, myocardial ischemia/reperfusion; ANI, anisodamine.
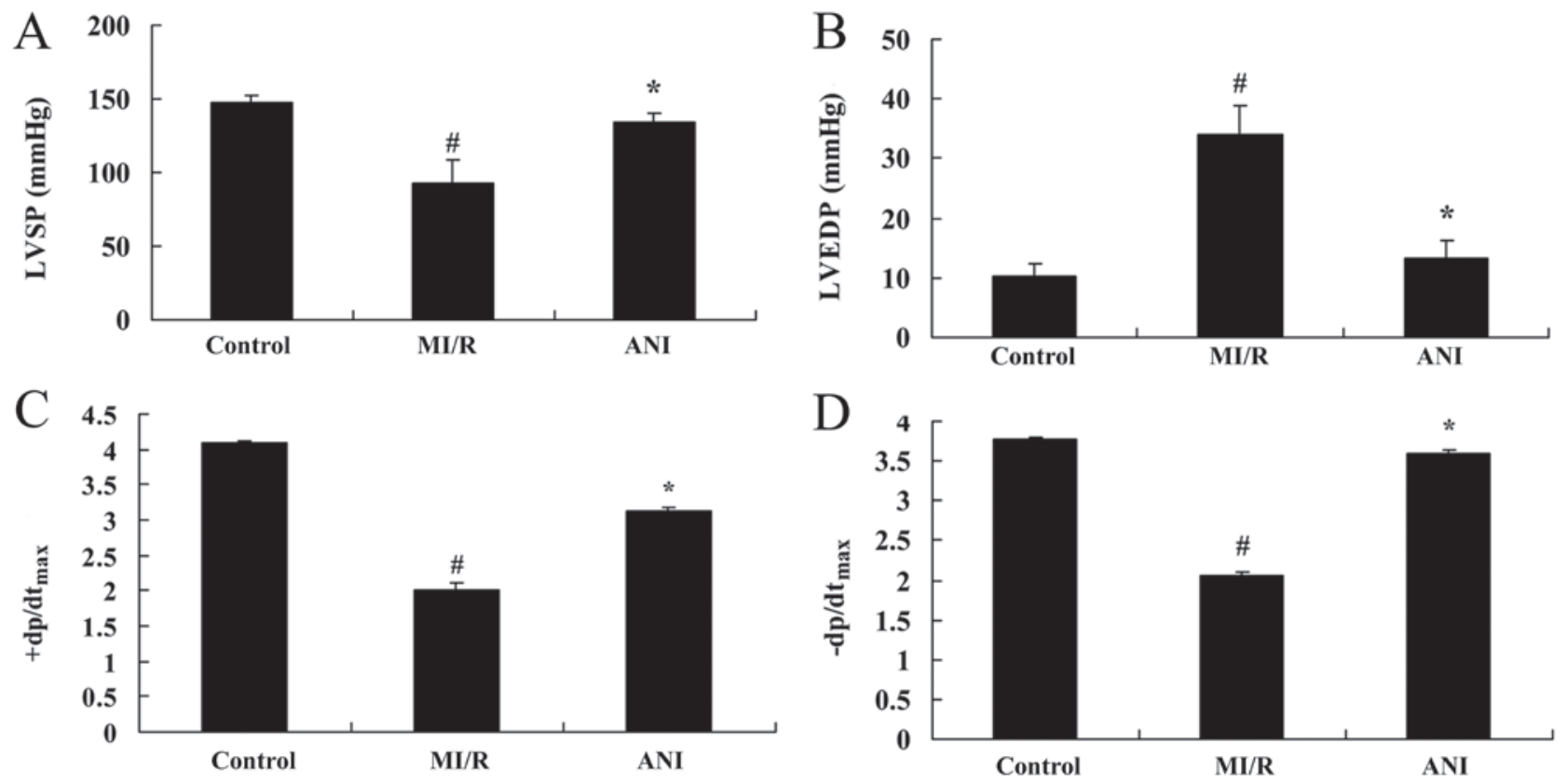

Figure 3. Effects of anisodamine on cardiac function. Treatment with anisodamine attenuated the I/R/-induced alterations in (A) LVSP, (B) LVEDP, (C) $+\mathrm{dp} / \mathrm{dt}$ max and (D) $-\mathrm{dp} / \mathrm{dt}_{\max }$ compared with rats in the I/R model group. Data are expressed as the mean \pm standard deviation. ${ }^{\#} \mathrm{P}<0.05$ vs. the control group; ${ }^{*} \mathrm{P}<0.05$ vs. the MI/R group. MI/R, myocardial ischemia/reperfusion; LV, left ventricular systolic pressure; LVEDP, left ventricular end-diastolic pressure; +dp/dt ${ }_{\text {max }}$, left ventricular pressure maximum rising rate; - $\mathrm{dp} / \mathrm{dt}_{\max }$, left ventricular pressure maximum falling rate; ANI, anisodamine.

A

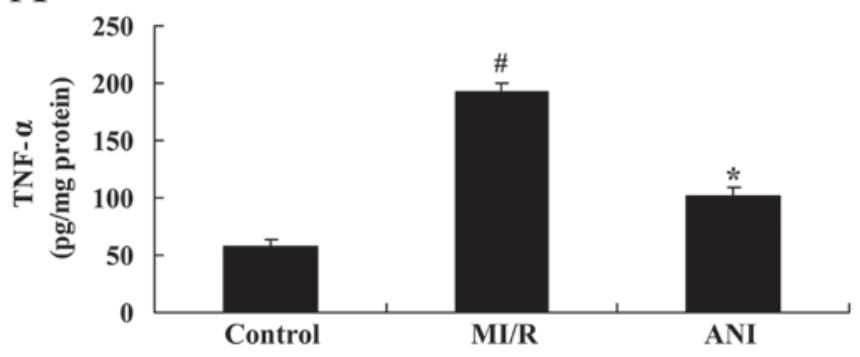

B

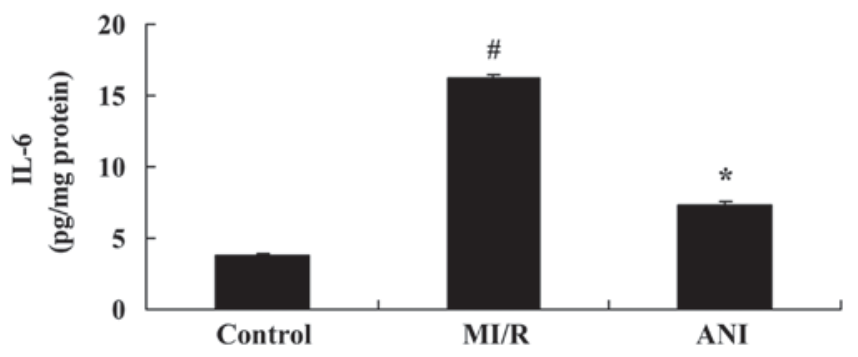

Figure 4. Effects of anisodamine on the production of inflammatory factors. Treatment with anisodamine significantly attenuated the I/R-induced increase in the serum levels of (A) TNF- $\alpha$ and (B) IL- 6 compared with the I/R model group. Data are expressed as the mean \pm standard deviation. ${ }^{\#} \mathrm{P}<0.05$ vs. the control group; *P<0.05 vs. the MI/R group. MI/R, myocardial ischemia/reperfusion; TNF, tumor necrosis factor; IL, interleukin; ANI, anisodamine.

anisodamine resulted in a significant downregulation in the $\mathrm{Bax} / \mathrm{Bcl}-2$ expression ratio compared with in the untreated $\mathrm{I} / \mathrm{R}$ model rats (Fig. 8).
Effects of anisodamine on iNOS and eNOS protein expression. The protein expression levels of iNOS and eNOS in rats following myocardial I/R were assessed using western blot analysis (Fig. 9). 
A

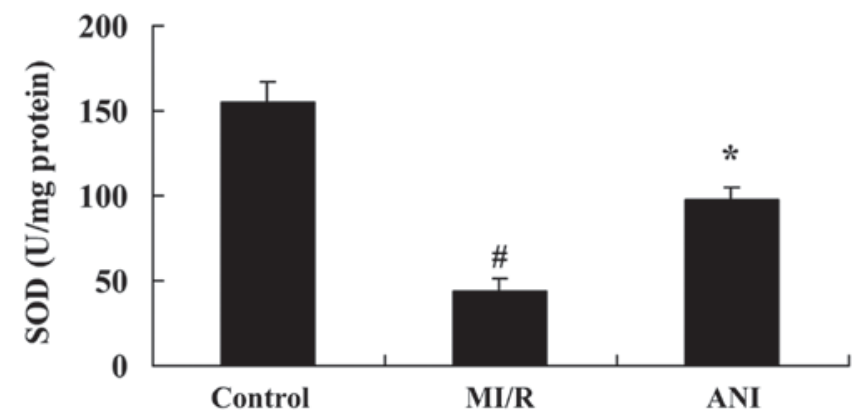

$\mathrm{B}$

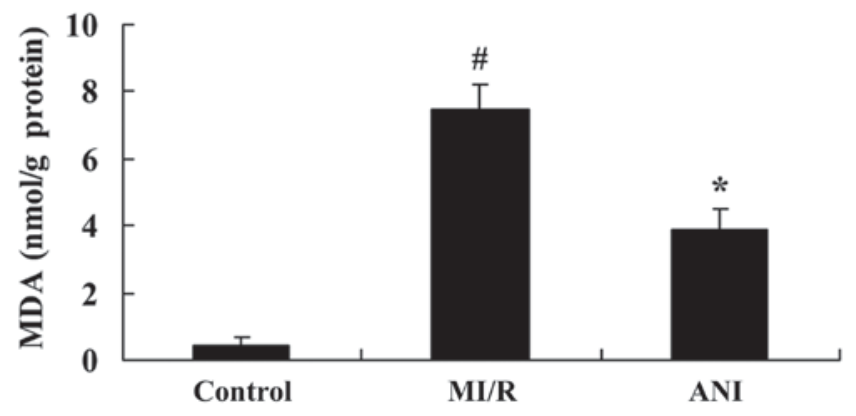

Figure 5. Effects of anisodamine on oxidative stress. Treatment with anisodamine reversed the I/R-induced alterations in (A) SOD and (B) MDA serum levels compared with the I/R model group. Data are expressed as the mean \pm standard deviation. ${ }^{\#} \mathrm{P}<0.05$ vs. the control group; $\mathrm{P}<0.05$ vs. the $\mathrm{MI} / \mathrm{R}$ group. $\mathrm{MI} / \mathrm{R}$, myocardial ischemia/reperfusion; SOD, superoxide dismutase; MDA, malondialdehyde; ANI, anisodamine.

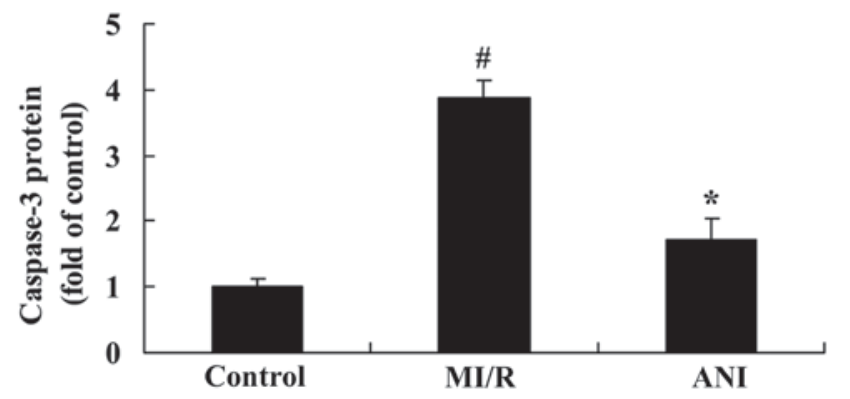

Figure 6. Effects of anisodamine on caspase-3 serum levels. Treatment with anisodamine significantly suppressed the I/R-induced increase in caspase-3 serum levels compared with the I/R model group. Data are expressed as the mean \pm standard deviation. ${ }^{\#} \mathrm{P}<0.05$ vs. the control group; ${ }^{*} \mathrm{P}<0.05$ vs. the $\mathrm{MI} / \mathrm{R}$ group. MI/R, myocardial ischemia/reperfusion; ANI, anisodamine.

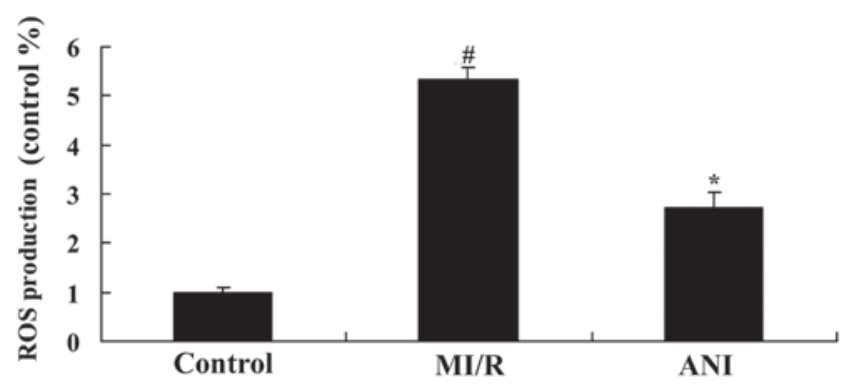

Figure 7. Effects of anisodamine on ROS generation. Treatment with anisodamine significantly suppressed the I/R-induced increase in ROS generation compared with the I/R model group. Data are expressed as the mean \pm standard deviation. " $\mathrm{P}<0.05$ vs. the control group; ${ }^{*} \mathrm{P}<0.05$ vs. the $\mathrm{MI} / \mathrm{R}$ group. ROS, reactive oxygen species; MI/R, myocardial ischemia/reperfusion; ANI, anisodamine.

Compared with the control group, a significant upregulation in iNOS and a downregulation in eNOS protein expression was detected in myocardial tissue samples from I/R rats (Fig. 9A, $\mathrm{B}$ and D). Treatment with anisodamine was demonstrated to significantly inhibit the protein expression of iNOS and induce the protein expression of eNOS following I/R compared with in untreated rats from the I/R model group (Fig. 9A, B and D).

Effects of anisodamine on Nox4 protein expression and Nox activity. Following myocardial I/R in vivo, the protein expression levels of Nox4 were significantly upregulated and the activity of Nox was significantly potentiated compared with in rats from the control group (Fig. 9C and E). Notably, treatment with anisodamine appeared to suppress the I/R-induced alterations. Western blot analysis revealed anisodamine administration significantly downregulated Nox4 protein expression, and Nox activity was also suppressed compared with in untreated I/R rats (Fig. 9C and E).

\section{Discussion}

During myocardial I/R injury, the intracellular concentration of $\mathrm{Ca}^{2+}$ is abnormally increased, resulting in the induction of myocyte apoptosis (17). Intracellular $\mathrm{Ca}^{2+}$ overload promotes the generation of oxygen free radicals, which can damage organelle membranes, enhance intracellular acidosis and impair the mitochondrial electron transport chain. In addition, proteases, lipases and nucleases are activated, and cause plasma membrane damage, lysis of structural proteins and chromosomal damage, resulting in impaired cellular metabolism and function, which promotes I/R-induced injury (18). The results of the present study demonstrated that treatment with anisodamine attenuated myocardial infarct sizes, decreased serum CK and LDH levels, and improved cardiac function parameters, including LVSP, LVEDP, $+\mathrm{dp} / \mathrm{dt}_{\max }$ and $-\mathrm{dp} / \mathrm{dt}_{\max }$, in rats following the induction of $\mathrm{I} / \mathrm{R}$ injury.

During myocardial I/R injury, the complement system is activated, which increases the production of adherence factors and the secretion of chemotactic factors (19). Inflammatory mediators, including leukotrienes, are attracted through chemotaxis, to ischemic tissues, and PMNs are recruited and activated in the site of injury. During reperfusion, ROS generation is potentiated in cardiac muscle tissue (9). Activated hemamebas adhere, transform and deposit in blocked myocardiac microvessels, causing neighboring cells to enter a hypoxic state and impacting cellular metabolism (20). Activated white blood cells in the lumen of blood vessels attach to the endothelium, where they synthesize and release vasoactive substances and inflammatory mediators, thus increasing vascular permeability, triggering inflammatory responses, and ultimately contributing to endothelial and cardiomyocyte damage (20). The present study demonstrated that treatment with anisodamine significantly suppressed the I/R-induced 
A

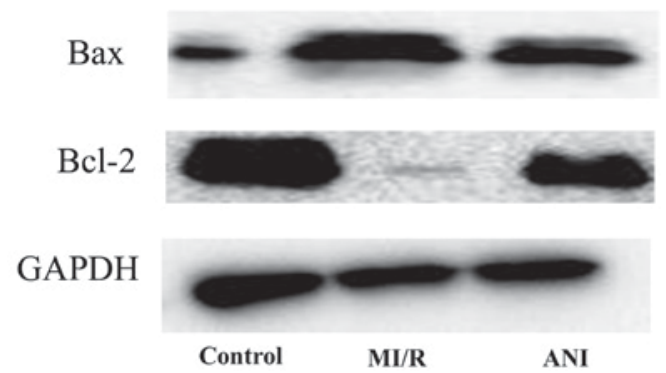

B

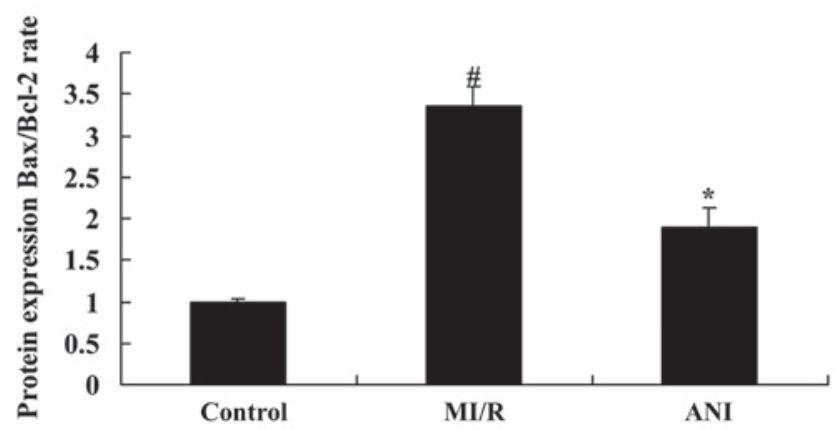

Figure 8. Effects of anisodamine on the expression of apoptosis-related proteins. (A) Western blot analysis was used to assess the protein expression levels of Bcl-2 and Bax. (B) Quantification of the western blotting results. Data are expressed as the mean \pm standard deviation. ${ }^{\#} \mathrm{P}<0.05$ vs. the control group; ${ }^{*} \mathrm{P}<0.05$ vs. the MI/R group. Bcl-2, apoptosis regulator Bcl-2; Bax, apoptosis regulator BAX; MI/R, myocardial ischemia/reperfusion; ANI, anisodamine.
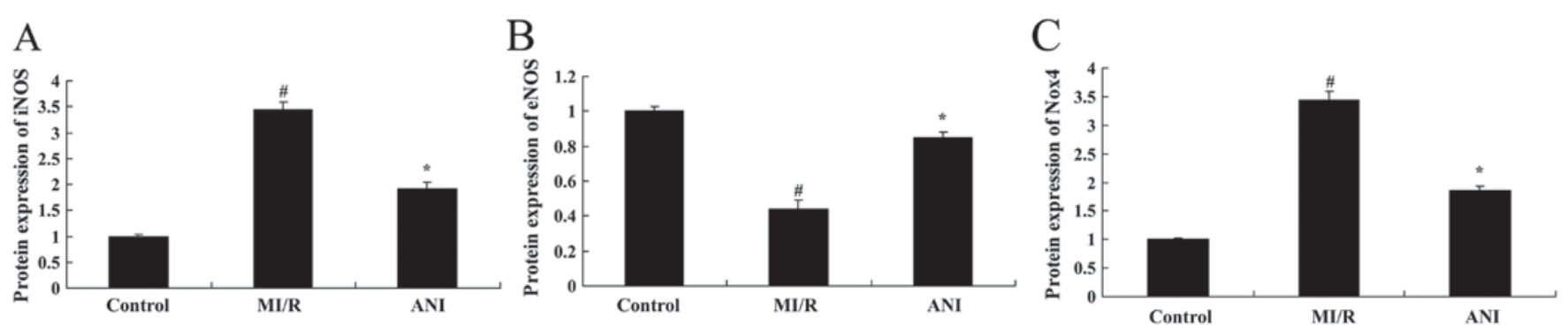

$\mathrm{D}$
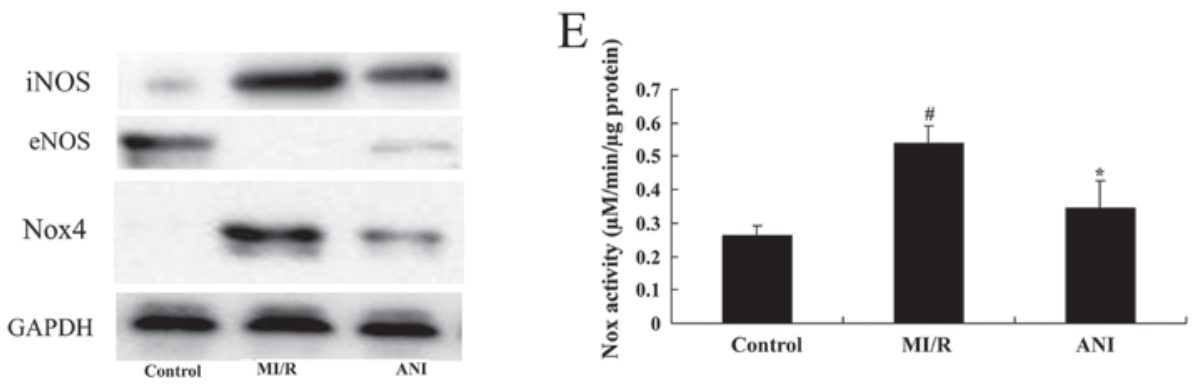

Figure 9. Effects of anisodamine on iNOS, eNOS and Nox4 protein expression, and Nox activity. The effects of anisodamine on iNOS and eNOS protein expression levels were assessed using western blot analysis. (A) Treatment with anisodamine significantly inhibited the protein expression of iNOS compared with in rats from the I/R model group. (B) Treatment with anisodamine significantly induced the protein expression of eNOS compared with in rats from the I/R model group. (C) Quantified protein expression levels of Nox4 following western blot analysis. (D) Representative blot demonstrating the expression of iNOS, eNOS and Nox4 in rats from the control, MI/R and anisodamine groups. (E) Treatment with anisodamine significantly suppressed the activity of Nox compared with in untreated I/R rats. Data are expressed as the mean \pm standard deviation. ${ }^{*} \mathrm{P}<0.05$ vs. the control group; ${ }^{*} \mathrm{P}<0.05$ vs. the $\mathrm{MI} / \mathrm{R}$ group. iNOS, inducible nitric oxide synthase; eNOS, endothelial NOS; MI/R, myocardial ischemia/reperfusion; ANI, anisodamine; Nox, nicotinamide-adenine dinucleotide phosphate oxidase.

increase in TNF- $\alpha$ and IL-6 serum levels in rats following the induction of I/R injury. In accordance with the present results, $\mathrm{Xu}$ et al (21) reported that anisodamine suppressed $\mathrm{T}$ helper cell type 2-associated responses and eosinophil-mediated inflammatory processes in a murine model of allergic asthma.

The mitochondrial electron transport chain and Nox are primarily responsible for ROS generation under physiological conditions. During myocardial I/R injury, ROS production from the mitochondria and Nox is implicated in oxidative stress injury in cardiomyocytes (22). Nox is an enzyme also expressed in phagocytes; however, Nox in myocardial cells differs in its catalytic characteristics and biological functions: Cardiac muscle Nox is weakly active under physiological conditions, whereas ROS production is a critical function of phagocytosis; cardiac Nox and NADPH or nicotinamide adenine dinucleotide (NADH) are electron donors, whereas only NADPH is an electron donor in phagocytes; in phagocytes, Nox-generated ROS mainly participate in the host mechanisms of defense $(23,24)$, whereas in cardiomyocytes ROS function as a second messenger during the regulation of cellular proliferation and differentiation (22). The results of the present study revealed that anisodamine significantly reversed the I/R-induced decrease in SOD levels and increase in MDA levels in serum samples isolated from rats following I/R. In accordance with the present results, Liu et al (25) reported that anisodamine attenuated oxidative stress-induced mitochondrial injury in swine with cardiac arrest.

Nox4 exhibits characteristics that distinguish it from other members of the Nox family of enzymes: Nox 2 and Nox 1 share a high sequence homology, whereas the amino acid sequence of Nox4 exhibits only a $39 \%$ homology with other Nox members, suggesting a corresponding difference in Nox4 structure. In 
addition, under physiological conditions, Nox4 activity is independent of regulatory subunits and is constitutively active; the activity of Nox4 is mainly controlled by its expression levels. Due to differences in their activation mechanisms, Nox2 needs to be induced in order to generate $\mathrm{O}^{2-}$, whereas Nox4 can constitutively generate low levels of $\mathrm{H}_{2} \mathrm{O}_{2}(26)$. Furthermore, Nox4 in cardiac muscle cells can catalyze $\mathrm{O}^{2-}$ generation effectively, using NADH as a hydrogen donor (23). Due to the autonomous activity of Nox4, NADH is highly used in cardiac muscle cells (26). The results of the present study demonstrated that anisodamine significantly suppressed the I/R-induced upregulation in Nox4 protein expression and Nox activity in $\mathrm{I} / \mathrm{R}$ model rats in vivo.

ROS and ROS-mediated oxidative stress responses have been implicated in cardiac hypertrophy induced by $\alpha$-adrenergic agonists, angiotensin II, endothelin (ET)-1 and TNF- $\alpha$ (23). Nox 2 and Nox4 in cardiac muscle cells have also been reported to participate in the development and progression of cardiac hypertrophy and fibrosis (18). Nox2 has been demonstrated to exacerbate hypertension triggered by cardiac hypertrophy, interstitial fibrosis and aldosterone/salt overload, whereas the expression of Nox4 can increase following stimulation by angiotensin II, $\alpha$-adrenergic agonists and hypertension (18). Nox4 is the main source of ROS in the hypertrophic heart (23). The results of the present study demonstrated that treatment with anisodamine significantly attenuated the I/R-induced increase in ROS generation in rat myocardial tissue in vivo.

Under physiological conditions, NO is released by the vascular endothelium and serves an important protective role for endothelial function (27). ET contents in the circulation are low under physiological conditions, and its effects on the endothelium are negligible (27). During I/R injury, the endothelial synthesis and release of NO is reduced, weakening antagonistic oxygen radicals, and its protective effects on the endothelium are lost, thus further aggravating vascular endothelial injury and forming a vicious cycle during the pathogenesis of I/R injury (28). Previous studies have reported that during myocardial I/R, the plasma NO concentrations are reduced and the ET concentrations are increased $(27,28)$. In the present study, anisodamine was revealed to significantly downregulate the protein expression of iNOS and upregulate the expression of eNOS, thus counteracting the I/R-induced dysfunctions in NO production in vivo.

In conclusion, the present study demonstrated that anisodamine exerted cardioprotective effects against myocardial I/R injury, through the inhibition of oxidative stress, inflammation and apoptosis, via targeting the expression of NOS and Nox, and the production of ROS. The present results suggested that anisodamine may have potential as an alternative therapeutic strategy for the treatment of patients with myocardial infarction.

\section{References}

1. Woodcock A, Bakerly ND, New JP, Gibson JM, Wu W, Vestbo J and Leather D: The salford lung study protocol: A pragmatic, randomised phase III real-world effectiveness trial in asthma. BMC Pulm Med 15: 160, 2015.

2. Gafarov VV, Panov DO, Gromova EA, Gagulin IV and Gafarova AV: Workplace stress and its impact on the 16-year risk of myocardial infarction and stroke in an open female population aged 25-64 years in Russia/Siberia (WHO MONICA-psychosocial program). Ter Arkh 87: 71-76, 2015.
3. Horspool MJ, Julious SA, Boote J, Bradburn MJ, Cooper CL, Davis S, Elphick H, Norman P, Smithson WH and VanStaa T: Preventing and lessening exacerbations of asthma in school-age children associated with a new term (PLEASANT): Study protocol for a cluster randomised control trial. Trials 14: 297, 2013.

4. Kim DE, Lee Y, Kim M, Lee S, Jon S and Lee SH: Bilirubin nanoparticles ameliorate allergic lung inflammation in a mouse model of asthma. Biomaterials 140: 37-44, 2017.

5. Chen YP, Zhang JH, Li CQ, Sun QX and Jiang XH: Obesity enhances Th2 inflammatory response via natural killer T cells in a murine model of allergic asthma. Int J Clin Exp Med 8: 15403-15412, 2015.

6. Zambalde ÉP, Teixeira MM, Favarin DC, de Oliveira JR, Magalhães ML, Cunha MM, Silva WC Junior, Okuma $\mathrm{CH}$, Rodrigues V Junior, Levy BD and Rogerio AP: The anti-inflammatory and pro-resolution effects of aspirin-triggered RvD1 (AT-RvD1) on peripheral blood mononuclear cells from patients with severe asthma. Int Immunopharmacol 35: 142-148, 2016.

7. Cao L, Guo C, Chen J, Chen Z and Yan Z: Free vascularized fibular grafting improves vascularity compared with core decompression in femoral head osteonecrosis: A randomized clinical trial. Clin Orthop Relat Res 475: 2230-2240, 2017.

8. Celik O, Celik E, Turkcuoglu I, Yilmaz E, Ulas M, Simsek Y, Karaer A, Celik N, Aydin NE, Ozerol I and Unlu C: Surgical removal of endometrioma decreases the NF-kB1 (p50/105) and NF-kB p65 (Rel A) expression in the eutopic endometrium during the implantation window. Reprod Sci 20: 762-770, 2013.

9. Wang W, Yu JN and Tao XJ: Systemic lupus erythematosus complicated with femoral head ischemic necrosis treated by Chinese medicine therapy for activating blood and dredging collaterals method. Chin J Integr Med 17: 105-110, 2011.

10. Wei L, Zhang H, Li X, Yang C, Wang G, Zhang L, Cui M and Han L: Efficacy and safety evaluation of intravenous infusion of cervus and cucumis polypeptides for treatment of avascular necrosis of the femoral head: A randomized clinical trial. J Tradit Chin Med 36: 39-44, 2016.

11. Fan JB, Ruan JW, Liu W, Zhu LQ, Zhu XH, Yi H, Cui SY, Zhao JN and Cui ZM: miR-135b expression downregulates Ppmle to activate AMPK signaling and protect osteoblastic cells from dexamethasone. Oncotarget 7: 70613-70622, 2016.

12. Jia J, Feng X, Xu W, Yang S, Zhang Q, Liu X, Feng Y and Dai Z: MiR-17-5p modulates osteoblastic differentiation and cell proliferation by targeting SMAD7 in non-traumatic osteonecrosis. Exp Mol Med 46: e107, 2014.

13. Yuan HF, Christina VR, Guo CA, Chu YW, Liu RH and Yan ZQ: Involvement of MicroRNA-210 demethylation in steroid-associated osteonecrosis of the femoral head. Sci Rep 6: 20046, 2016.

14. Song Y, Du Z, Ren M, Yang Q, Wang Q, Chen G, Zhao H, Li Z, Wang J and Zhang G: Association of gene variants of transcription factors PPAR $\gamma$, RUNX2, Osterix genes and COL2A1, IGFBP3 genes with the development of osteonecrosis of the femoral head in Chinese population. Bone 101: 104-112, 2017.

15. Wan P, Su W, Zhang Y, Li Z, Deng C and Zhuo Y: Trimetazidine protects retinal ganglion cells from acute glaucoma via the Nrf2/Ho-1 pathway. Clin Sci (Lond) 131: 2363-2375, 2017.

16. Wang XR, Shi GX, Yang JW, Yan CQ, Lin LT, Du SQ, Zhu W, He T, Zeng XH, Xu Q and Liu CZ: Acupuncture ameliorates cognitive impairment and hippocampus neuronal loss in experimental vascular dementia through Nrf2-mediated antioxidant response. Free Radic Biol Med 89: 1077-1084, 2015.

17. Milger K, Götschke J, Krause L, Nathan P, Alessandrini F, Tufman A, Fischer R, Bartel S, Theis FJ, Behr J, et al: Identification of a plasma miRNA biomarker signature for allergic asthma: A translational approach. Allergy: May 17, 2017 (Epub ahead of print).

18. Zhou ZC, Gu SZ, Wu J and Liang QW: VEGF, eNOS, and ABCB1 genetic polymorphisms may increase the risk of osteonecrosis of the femoral head. Genet Mol Res 14: 13688-13698, 2015.

19. Jonsson BA, Kadar T, Havelin LI, Haugan K, Espehaug B, Indrekvam K, Furnes $\mathrm{O}$ and Hallan G: Oxinium modular femoral heads do not reduce polyethylene wear in cemented total hip arthroplasty at five years: A randomised trial of 120 hips using radiostereometric analysis. Bone Joint J 97-B: 1463-1469, 2015.

20. Yun SJ, Nam DH and Ryu JK: Femoral artery access using the US-determined inguinal ligament and femoral head as reliable landmarks: Prospective study of usefulness and safety. J Vasc Interv Radiol 26: 552-559, 2015.

21. Xu ZP, Wang H, Hou LN, et al: Modulatory effect of anisodamine on airway hyper-reactivity and eosinophilic inflammation in a murine model of allergic asthma. Int Immunopharmacol 11: 260-265, 2011. 
22. Tingart M, Beckmann J, Opolka A, Matsuura M, Schaumburger J, Grifka J and Grässel S: Analysis of bone matrix composition and trabecular microarchitecture of the femoral metaphysis in patients with osteonecrosis of the femoral head. J Orthop Res 27: $1175-1181,2009$.

23. Kong X, Li X, Zhang C, Zhu L, Liu C, Qin Q, Liu C, Wang Q, Zhu J, Wu X, et al: Ethyl acetate fraction of Huogu formula inhibits adipogenic differentiation of bone marrow stromal cells via the BMP and Wnt signaling pathways. Int J Biol Sci 13: 480-491, 2017.

24. Lin D, Zuo S, Li L, Wang L and Lian K: Treatment of neglected femoral neck fractures using the modified dynamic hip screw with autogenous bone and bone morphogenetic protein-2 composite materials grafting. Indian J Orthop 49: 342-346, 2015.

25. Liu YH, Zhang J, Dai Z, et al: [Protection of anisodamine on the mitochondrial injury induced by oxidative stress in swine with cardiac arrest]. Zhonghua Wei Zhong Bing Ji Jiu Yi Xue 25: 290-293, 2013
26. Renkawitz T, Santori FS, Grifka J, Valverde C, Morlock MM and Learmonth ID: A new short uncemented, proximally fixed anatomic femoral implant with a prominent lateral flare: Design rationals and study design of an international clinical trial. BMC Musculoskelet Disord 9: 147, 2008.

27. Ma XL, Liu ZP, Ma JX, Han C and Zang JC: Dynamic expression of Runx2, Osterix and AJ18 in the femoral head of steroid-induced osteonecrosis in rats. Orthop Surg 2: 278-284, 2010.

28. Li J, Wang Y, Li Y, Sun J and Zhao G: The effect of combined regulation of the expression of peroxisome proliferator-activated receptor- $\gamma$ and calcitonin gene-related peptide on alcohol-induced adipogenic differentiation of bone marrow mesenchymal stem cells. Mol Cell Biochem 392: 39-48, 2014. 